\title{
Circulating molecular biomarkers for screening or early diagnosis of colorectal cancer: which is ready for prime time?
}

\author{
Elisa Danese", Martina Montagnana", Giuseppe Lippi \\ Section of Clinical Biochemistry, University of Verona, Verona, Italy \\ Contributions: (I) Conception and design: All authors; (II) Administrative support: None; (III) Provision of study materials or patients: None; (IV) \\ Collection and assembly of data: All authors; (V) Data analysis and interpretation: All authors; (VI) Manuscript writing: All authors; (VII) Final \\ approval of manuscript: All authors. \\ "These authors contributed equally to this work. \\ Correspondence to: Prof. Giuseppe Lippi. Section of Clinical Biochemistry, University Hospital of Verona, Piazzale LA Scuro, Verona 37134, Italy. \\ Email: giuseppe.lippi@univr.it.
}

\begin{abstract}
According to recent statistics, colorectal cancer (CRC) is a frequent disease, the second most frequent malignancy in women and the third most common malignant disease in men, respectively. Although reinforced emphasis on CRC screening by means of immunochemical fecal occult blood test, colonoscopy or sigmoidoscopy has contributed to decrease cancer-related deaths, alternative diagnostic tests would be needed for establishing earlier and more potentially effective treatments. Innovative diagnostic techniques have recently emerged, some of which hold promises for screening and/or early CRC detection. Recent evidence suggests that the so-called "liquid biopsy", conventionally defined as detection and quantification of circulating tumor cells (CTCs) and cancer-related nucleic acids in peripheral blood, may allow earlier diagnosis of CRC combined with lower invasiveness and less patient inconvenience, higher throughput, faster turnaround time, inferior usage of healthcare resources and relatively low cost. Encouraging data have emerged from trials based on CTCs detection, though the sensitivity of the current diagnostic techniques is still perhaps insufficient for enabling early CRC diagnosis. Among the various biomarkers that can be detected with liquid biopsy, SEPT9 methylation displays good diagnostic performance and relatively high cancer detection rate (between $57-64 \%$ in patients with CRC stages 0-I), which would make this test a promising tool for population screening, alone or in combination with other conventional diagnostic investigations. Encouraging evidence has also been recently published for BCAT1/IKZF1 methylation. Regarding microRNA (miRNAs), the available evidence highlights that the combination of some of these biomarkers rather than the assessment of a single miRNA alone would enable efficient identification of early CRCs, though widespread clinical application is still challenged by a number of preanalytical, analytical and clinical issues.
\end{abstract}

Keywords: Colorectal cancer (CRC); circulating tumor DNA (ctDNA); circulating tumor cells (CTCs); early diagnosis; microRNAs

Submitted Aug 19, 2019. Accepted for publication Aug 21, 2019.

doi: $10.21037 /$ atm.2019.08.97

View this article at: http://dx.doi.org/10.21037/atm.2019.08.97

\section{State-of-the-art in colorectal cancer (CRC) screening}

CRC is a very frequent disease, accounting for as many as 1.85 million of new cases each year, and thus being currently considered the second most frequent malignancy in women after breast cancer and the third most common malignant disease in men after lung and prostate cancers (1). Several lines of evidence now attest that the prognosis of this cancer is highly dependent on early diagnosis, whereby the 5-year cumulative survival rate decreases from $90 \%$ when CRC 
is diagnosed at an early stage, to $\sim 15 \%$ in patients with advanced, metastatic disease (1).

Although reinforced emphasis placed on CRC screening has indeed contributed to ameliorate its otherwise unfavorable biologic course, the vast majority of patients with this malignant disease can only be diagnosed after symptoms onset, when cancer growth has already seriously jeopardized treatment success and life expectancy $(2,3)$. Despite its well-known, inherent limitations (4), the immunochemical fecal occult blood test (iFOBT) is still considered the most cost-effective analysis for CRC screening around the world. The widespread use of this test is endorsed (in people aged 45 years or older) by the American Cancer Society (ACS) (5). Rather similar indications are provided by other national and international scientific organizations, which are almost concordant to agree on the cost-effectiveness of screening subjects aged between 50-75 years, by means of iFOBT, colonoscopy or flexible sigmoidoscopy (6).

The evidence that iFOBT screening contributes to lower the overall number of deaths for CRC is now generally taken for granted, though recent evidence seems to contradict this widespread perception, at least for what concerns all-cause deaths and certain cancer sites. Shaukat et al. for example, recently published the results of the Minnesota Colon Cancer Control Study, in which over 46,000 participants aged between $50-80$ years were randomized to receive either no screening or annual or biennial iFOBT testing (2). At the end of 30-year followup, colorectal-cancer mortality was reduced by $~ 30 \%$ with annual screening [relative risk (RR), $0.68 ; 95 \%$ confidence interval (95\% CI), 0.56-0.82] and $20 \%$ with biennial screening (RR, 0.78; 95\% CI, 0.65-0.93), but no significant decline was noted for all-cause mortality (annual screening; RR, 1.00 and 95\% CI, 0.99-1.01; biennial screening: RR, 0.99; 95\% CI, 0.98-1.01). Most notably, similar results were nearly concomitantly published by Nishihara et al., who explored the effectiveness of endoscopy for preventing CRC mortality in over 88,000 participants of the Nurses' Health Study and the Health Professionals Follow-up Study (7). At the end of 22-year follow-up, CRC mortality was considerably reduced by $\sim 40 \%$ [hazard ratio (HR), 0.59; 95\% CI, 0.45-0.76] with screening sigmoidoscopy and by $~ 70 \%$ (HR, $0.32 ; 95 \%$ CI, $0.24-0.45$ ) with screening colonoscopy, respectively. Nevertheless, a sub-analysis of the data revealed that screening sigmoidoscopy was ineffective to lower the number of deaths due to proximal colon cancer (HR, 1.04; 95\% CI, 0.73-1.48).
Importantly, Welch and Robertson have recently highlighted that the decline of all-cause and cancer-related mortality recently observed in patients with colorectal malignancies cannot be entirely explained by adherence to population screening, whereby multiple other factors may have played a role (8). Therefore, even without openly denying the unquestionable effectiveness of strategies currently used for CRC screening (i.e., iFOBT, colonoscopy or sigmoidoscopy), the still elevated mortality for CRC and the significant risk of false negative test results, lead the way to developing alternative diagnostic tests, which may allow to establish an earlier and thus more potentially effective treatment (9). Innovative diagnostic techniques have recently been proposed, such as video capsule endoscopy, liquid biopsy, analysis of multitarget-stool DNA or analysis of volatile organic compounds in exhaled air $(10,11)$, but none of these approaches seems ready for prime time. Nevertheless, encouraging data are emerging for some molecular blood biomarkers, whose clinical usefulness will be discussed in the following parts of this article.

\section{Liquid biopsy: current evidence and future perspectives}

The blood of patients with different forms of cancer may theoretically contain a variable number of circulating tumor cells (CTCs), cell-derived vesicles (such as exosomes) and cell-free nucleic acids released from the tumor itself. The term "liquid biopsy" was originally used for identifying CTCs in peripheral blood, but was then extended to detection and quantification of circulating tumor DNA (ctDNA) and microRNAs (miRNAs). Even if bloodbased assays are those most widely used, ctDNA could be detected in many other biological fluids such as pleural effusion, ascites, saliva, urine, stool and cerebrospinal fluid. Therefore, the term "liquid biopsy" is now used for characterizing a broad set of tests used for analysis of tumor-derived biomarkers isolated from biological fluids of cancer patients (12).

Since CRC is often the worse consequence of evolving precancerous lesions (e.g., adenomas), screening is mostly focused on early detection of these pathologies. The use of liquid biopsy not only would be useful for early diagnosing CRC, but also may carry many other advantages, including lower invasiveness and less patient inconvenience (compared with colonoscopy and/or sigmoidoscopy), higher throughput, faster turnaround time, minor usage of healthcare resources and relatively low cost $(12,13)$. 
Since malignant cells follow a continuous and dynamic evolution in the bloodstream, paralleling cancer growth and spread, another advantage of liquid biopsy is the possibility to monitor cancer genome and molecular properties in real time, thus enabling rapid detection of somatic mutations that may impact the clinical course of colorectal malignancies (14).

Several clinical applications of liquid biopsy have been investigated in CRC, ranging from early disease detection to their usage as prognostic or predictive tools, for monitoring disease or for studying the mechanisms involved in development of drug resistance (15). Nevertheless, the use of this diagnostic strategy is still limited in clinical practice. The insufficient level of standardization for isolation, detection and quantification of nucleic acids is perhaps one of the most important causes for its narrow usage (16). Moreover, further prospective studies, including large sample sizes, would be needed to clearly demonstrate the clinical effectiveness or superiority of this technique over the current armamentarium of conventional diagnostic tests (17).

\section{CTCs}

CTCs are essentially tumor-derived cells, in particular epithelial cancer cells, which shed into peripheral blood by passive cell shedding or active regulated process (18). Epithelial-to-mesenchymal transition (EMT) is the highly dynamic process by which a sub-population of primary cancer cells acquires increased motility and invasiveness potential (19). CTCs have been identified in several types of cancers, as a single CTC or in aggregates ( $\geq 2$ CTCs), named "clusters" or "tumor microemboli" (20). It has been demonstrated that CTCs clusters are more prone to form metastases than single CTC (21), due to enhanced likelihood of "survival" (22). In fact, most CTCs released in the bloodstream early succumb for combined influence of mechanical and environmental injuries such as shear forces, oxidative stress and immune system response. The halflife of CTCs in the circulation is hence extremely narrow, typically comprised between 1-2 h (23).

In studies on breast cancer patients, some subset of CTCs with tumor-initiating capacity have been demonstrated $(24,25)$. Since the number of circulating CTCs is usually low, ranging between 1 and 10 cells per $10 \mathrm{~mL}$, perhaps even lower in early stage cancers (26), CTC enrichment and detection are two essential steps for accurate quantification. On the other hand, contamination is a major source of diagnostic problems, since most detection systems tend to capture also a variable number of leukocytes (12). CTCs enrichment can be achieved by assessing the expression of epithelial surface specific proteins or by targeting specific physical features such as size or density of cancer cells (27). As regards detection, several approaches (immunological, molecular and functional techniques) have been developed. Nevertheless, the only technology that has been approved so far by the US Food and Drug Administration (FDA) for CTC enumeration in patients with metastatic breast, prostate and CRC is CellSearch (Janssen Diagnostics, Raritan, NJ, USA), which is based on anti-epithelial cell adhesion molecule (EpCAM) antibodies (28).

What has clearly emerged from recent literature data is that the methods for enrichment, detection and characterization of CTCs lack sensitivity, specificity and reproducibility (29). Some technological breakthroughs hold promises, but they will need careful evaluation and verification before being implemented in routine clinical practice. Abouleila et al. have recently developed an interesting approach, based on use of live single cell mass spectrometry integrated with microfluidics-based cell enrichment, for assessing differences in metabolomic profile between CTCs originating from different cancer groups (30). Su et al. have also developed an integrated microfluidic chip device that is capable to easily and automatically enrich and identify CTCs from blood of CRC patients (31). Although these preliminary results seem promising (the enrichment efficiency for CTCs was reported to be as high as $70 \%$ ), further validation studies would be needed.

Although the prognostic value of CTC in early stages of CRC has already been proven by a large number of clinical studies $(17,32)$, their role for screening and early detection remains controversial (33). The still inefficient diagnostic performance has been principally attributed to evidence that the number of CRC patients who will test positive for CTCs in early stages of cancer is perhaps too low to achieve sufficient sensitivity with currently available techniques (15). Baek et al. have recently explored the clinical significance of CTCs assessment in early CRC detection (34). Although sensitivity and specificity for distinguishing CRC patients $(n=88)$ from a small cohort of healthy controls $(n=31)$ were $75 \%$ and $100 \%$, with a corresponding area under the curve (AUC) of 0.91, the authors did not carried out a sub-analysis for addressing the diagnostic performance of CTCs in early CRC stages (i.e., I and II). Nevertheless, encouraging results were published by Tsai and colleagues (35), who 
assessed CTCs in 182 healthy controls, 111 patients with precancerous lesions and 327 patients with stages I-IV CRC. The sensitivity, specificity and AUC of CTCs testing were $76.6 \%, 97.3 \%$ and 0.84 for differentiating patients with precancerous lesions from healthy subjects, and $86.9 \%$, $97.3 \%$ and 0.88 for distinguishing CRC patients from healthy subjects. Other studies will be obviously needed to validate the possible use of CTCs for early diagnosis of CRC.

\section{ctDNA}

\section{General considerations}

The term ctDNA is conventionally used for identifying the portion of circulating free DNA (cfDNA) comprised only by nucleic acid fragments originating from tumor cells. ctDNA is thought to principally originate from apoptosis and necrosis of cancer cells in tumor microenvironment (36), though active release by living and circulating cancer cells has also been suggested. The assessment of cfDNA integrity index (DII), defined as the ratio of longer to shorter DNA fragments, is often exploited as surrogate marker for distinguishing cfDNA released by necrosis or apoptosis. The rationale behind this assumption is that DNA originated from necrotic cells varies in size, whilst DNA released from apoptotic cells is uniformly truncated into fragments shorter than $200 \mathrm{bp}$. Although DII is frequently increased in patients with cancer, including those with $\mathrm{CRC}$, thus enlightening that necrosis may be the prevalent source of ctDNA, we and other groups have convincingly shown that predominance of one tumor cell mechanism over others may depend on specific cancer identity and also varies throughout cancer development (37).

Along with DII assessment, cfDNA can be used for detecting genetic and epigenetic CRC-specific abnormalities, whereby two of the most investigated ctDNA markers entail KRAS mutations and SEPT9 promoter methylation. In a study comparing data of a cfDNA assay with CRC tumor-tissue analysis we could demonstrate that both KRAS mutation analysis and SEPT9 methylation assay are characterized by a high degree of concordance in both blood and cancer tissue, thus supporting the hypothesis that cfDNA assessment may represent a valuable alternative to tissue analysis for both genetic and epigenetic tumor characterization (38). Interestingly, we also showed that transition of DNA harboring the epigenetic alteration into the circulation is more consistent than transition of DNA harboring a KRAS mutation in early CRC stages. Aberrant methylation of SEPT9 gene can thus be considered a sensitive and specific biomarker for early non-invasive diagnosis of CRC, which may theoretically be exploited for screening purposes. Unlike these findings, the overall sensitivity of KRAS ctDNA analysis for detecting CRC was found to be low, ranging between 3-63\%, and this has been explained with evidence that mutations in $K R A S$ may only be present in a minority of CRC patients (39). This preamble explains why KRAS status is mostly assessed for prognostic purposes, for monitoring tumor burden and for predicting chemo/immunotherapy response in CRC, but not for early diagnosing CRC. In particular KRAS status may predict response to established epidermal growth factor receptor (EGFR) inhibitors, whereby mutant KRAS is associated with resistance to anti-EGFR monoclonal antibody immunotherapy. An informative update on the role of SEPT9 methylation and other methylated genes in early CRC diagnosis is summarized in the follow paragraphs of this article.

\section{SEPT9 methylation}

The SEPT9 gene encodes a protein called septin-9, which is involved in a vast array of biological pathways such as cytoplasm division, cell polarization, vesicle transport and membrane reconstruction. This protein also acts as tumor suppressor, since it actively regulates cell growth and prevents uncontrolled cellular division. The methylation of SEPT9 at specific CpG islands in the promoter region is accompanied by gene silencing, with consequent loss of cancer suppressor activity. Due to the proven role of SEPT9 downregulation in pathological progression from benign to malignant lesions in colorectal tissue, the company Epigenomics AG (Frankfurt Prime Standard: ECX; Frankfurt, Germany) has commercialized the first bloodbased real-time PCR SEPT9 methylation assay nearly 10 years ago, which is available as commercial CE-IVD test throughout Europe with the name Epi proColon 1.0. (40). In 2011 the same company launched a second-generation test (Epi proColon 2.0), characterized by enhanced diagnostic performance and featuring some notable technical advancements (i.e., lower reagents, fewer handling steps and shorter turnaround time), which would be aimed at facilitating its use in routine diagnostics (41).

The SEPT9 methylation assay is an essentially qualitative test, which can be interpreted as positive or negative according to application of different algorithms. Positive test results can hence be defined by one positive count out of three PCRs (1/3 algorithm), one positive count out 
of two PCRs (1/2 algorithm), two positive counts out of three PCRs (2/3 algorithm), or one positive count out of one PCR (1/1 algorithm). To date, 25 independent trials, mostly case-control or cohort studies, have investigated the diagnostic performance of SEPT9 gene methylation assay for CRC detection by using either research assays or Epi proColon kits (42). The sensitivity and specificity of the assay were found to be largely dependent on study design, sample size, study population and type of test, cumulatively ranging between $48.2-95.6 \%$ and $79.1-99.1 \%$, respectively (42). Sixteen of these studies reported separated analyses for detection rate of SEPT9 methylation assay in polyps, adenoma and early stage cancers, as shown in Table 1 (43-58). As predictable, the highest detection rate was achieved for both stage I and stage II, by applying the $1 / 3$ algorithm. The overall diagnostic sensitivity of the $1 / 3$ algorithm ranged between $37.1-84.0 \%$ in stage I CRC, and between $57.1-100 \%$ in stage II CRC. The overall diagnostic sensitivity of the $2 / 3$ algorithm ranged between $17.1-66.7 \%$ in stage I CRC, and between $53.3-92.8 \%$ in stage II colorectal malignancies. The Prospective Evaluation of Septin 9 Performance for Colorectal Cancer Screening (PRESEPT) is the only screening study based on an average-risk population that has been performed so far. Song and coauthors compared data obtained in this study with those resulting from fecal immunochemical test (FIT) and FIT-DNA testing in the same population (42), demonstrating that the SEPT9 methylation assay displays lower diagnostic sensitivity (68.0\% versus $79.0 \%$ for FIT and $92.3 \%$ for FIT-DNA test, respectively) and lower diagnostic specificity $(80.0 \%$ versus $94.0 \%$ for FIT and $86.6 \%$ for FIT-DNA test, respectively) $(59,60)$. The diagnostic performance of blood SEPT9 assay in asymptomatic population screening was hence seemingly lower than that of both FIT and FIT-DNA tests. Nevertheless, the SEPT9 assay displayed a substantial better compliance than FIT testing.

Along with screening of average-risk population, the diagnostic performance of the SEPT9 assay was also investigated as part of opportunistic screening in highrisk population. In a large population study, including four northern Chinese hospitals and using the SensiColon test, SEPT9 assay displayed $76.6 \%$ sensitivity and $95.9 \%$ specificity for cancer identification, averaging a remarkable cancer detection rate as high as $25.8 \%$ in asymptomatic subjects (58). In another recent opportunistic screening study based on the Epi proColon 2.0 CE kit, SEPT9 assay displayed $75.1 \%$ sensitivity and $95.1 \%$ specificity for opportunistic CRC detection (55). Notably, the cancer detection rate was as high as $57 \%$ in CRC stage $0,64 \%$ in CRC stage I and $88 \%$ in CRC stage II, thus potentially making it a valuable tool for population screening, alone or in combination with other more conventional diagnostic investigations.

\section{Other methylated genes}

Beside SEPT9 methylation, which has unquestionably been the largest abnormality that has been investigated so far for screening and/or early diagnosis of CRC, the clinical significance of other methylated epigenetic biomarkers has been recently addressed.

In 2014, Mitchell and collaborators used a pipeline combining gene expression, targeted DNA methylation and genome-wide DNA methylation analyses for identifying a panel of methylated genes in CRC patients (61). Based on low background in plasma of normal subjects $(3.5 \%$ and $4.9 \%$ respectively), the two genes $B C A T 1$ and $I K Z F 1$ were found to be suitable for assay development and optimization, and were finally selected as putative biomarkers (62). In a 218-person case/control study, combined analysis of these two genes exhibited a 77\% cumulative diagnostic sensitivity for detecting CRC, associated with detection rate of $50 \%$ in stage I and $68 \%$ in stage II, respectively (63). The same combination of methylated genes was then assessed in a larger prospective clinical trial, including over 3,900 subjects (64). This study confirmed the highly positive detection rate in both stage I and II CRC (38\% and 69\%, respectively). In the same cohort of patients Symonds et al. showed that a test based on detecting methylated BCAT1/IKZF1 DNA in blood (positivity threshold of $10 \mu \mathrm{g} \mathrm{Hb} / \mathrm{g}$ ) displayed comparable diagnostic sensitivity (62\% versus $64 \%$ ) and specificity ( $92 \%$ for both) with FIT for early detecting CRC (65). In these studies, the diagnostic sensitivity of biomarkers combination for detecting colorectal adenomas was comprised between $6-9 \%(64,65)$.

In 2015 Lin et al. used a high-resolution, genome-wide approach for demonstrating that the promoters of $A G B L 4$, FLI1 and TWIST1 genes are hypermethylated in over $80 \%$ of CRCs, and that these epigenetic modifications could be reliably detected in plasma DNA samples. The sensitivity of any of the three genetic abnormalities in cfDNA for detecting CRC (including also stages I or II cancers) was as high as $90 \%$ (66). In another retrospective study, Lee et al. analyzed plasma samples of 243 patients with stage I and II sporadic CRC, 276 healthy individuals and 64 patients with colorectal adenomas using methylation- 


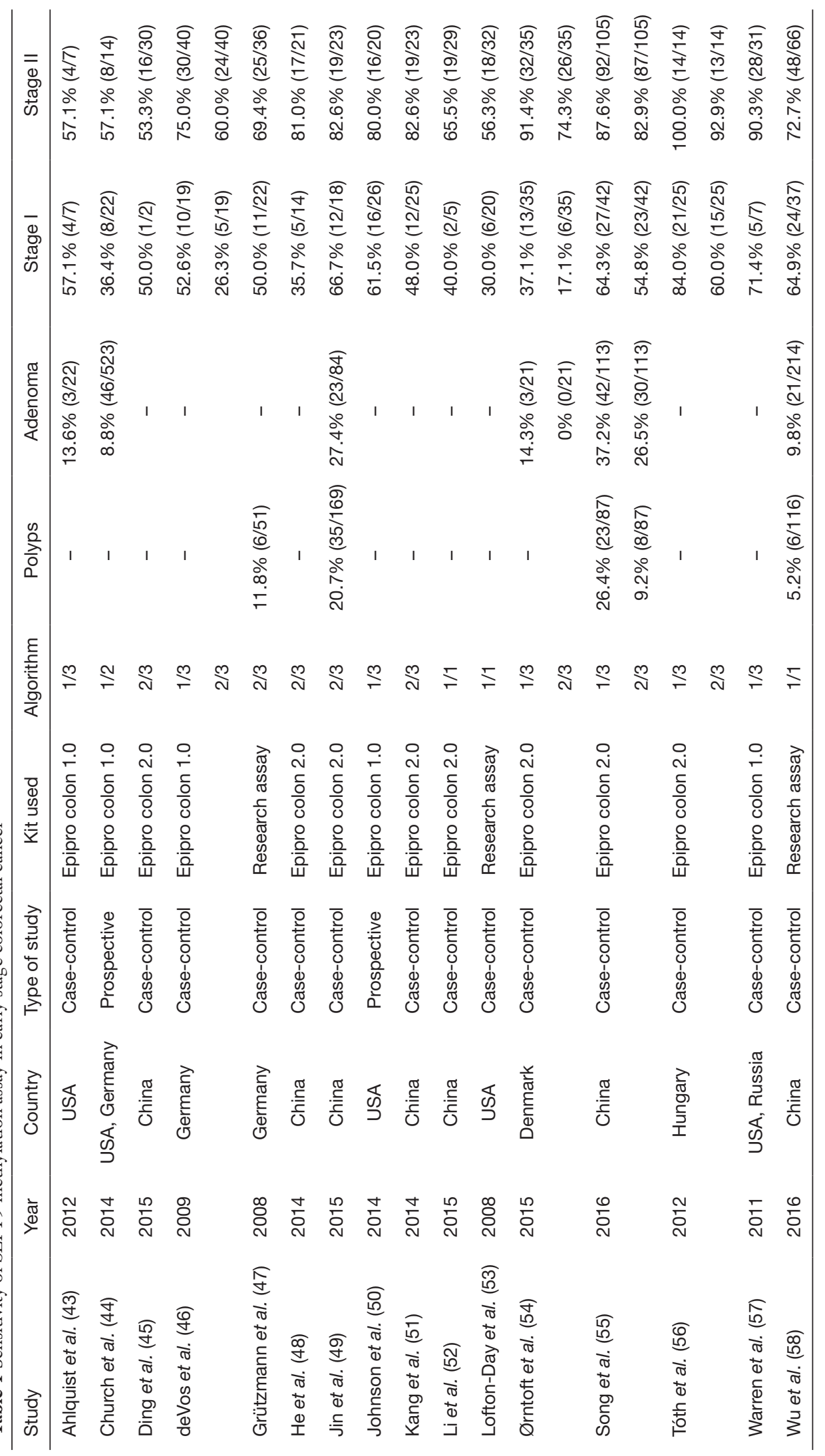


specific PCR (67). A panel of methylated genes, including $A P C, M G M T, R A S S F 2 A$ and $W i f-1$, displayed $86.5 \%$ diagnostic sensitivity and $92.1 \%$ diagnostic specificity. Notably, sensitivity was still as high as $86.5 \%$ for detecting early stage CRC, and approximated $75 \%$ for detecting adenomas. The detection rate of adenoma reported in this study is indeed the highest overall, and could not be replicated in other independent studies. SFRP2, ITGA4 and GATA5 are three additional methylated genes, for which some preliminary data were published in patients with colorectal adenoma. In a small case-control study, Zhang et al. reported a detection rate for precancerous lesions approximating $40 \%$ for SFRP2, $43 \%$ for GATA5 and $40 \%$ for ITGA4, respectively. The combination of SFRP 2 and GATA5 displayed acceptable predictive efficiency for detecting colorectal adenomas (odds ratio, 3.35; 95\% CI, 1.29-8.71) (68).

\section{Circulating miRNAs}

MiRNAs, small-non-coding single-strand stable RNAs containing approximately 20-24 nucleotides, play an essential role in post-transcriptional regulation of eukaryotic genes, so that they have become the most studied noncoding RNAs (ncRNAs) (69). These small molecules are actively involved in a kaleidoscope of both physiological and pathological cellular processes, thus including malignant transformation. Both plasma and serum are suitable sample matrices for investigating miRNAs expression $(70,71)$, though miRNAs can be theoretically assayed in all body fluids, since it is universally considered a relatively stable molecule (72). The leading techniques for studying miRNAs expression include quantitative reverse transcription-PCR (qRT-PCR), microarrays and next-generation sequencing (NGS) (73).

A number of meta-analyses have attempted to summarize the potential clinical usefulness of circulating miRNAs in early CRC detection (74-78), all converging to the conclusion that the diagnostic performance could be substantially magnified by simultaneously detecting more than a single miRNA.

In 2014, Wang and colleagues carried out a sub-analysis of 47 CRC studies (out of a total number of 107 studies in gastrointestinal malignancies), concluding that singlemiRNA assays (29 studies) had lower performance for diagnosing CRC compared to multiple-miRNAs assays (18 studies) (AUC, 0.79 versus 0.89) (74). Sensitivity, specificity and AUC were as high as $81 \%, 84 \%$ and 0.89 in the meta-analysis published by Zeng et al., which included 24 studies from 19 different articles, totaling 1,558 CRC patients and 1,085 controls (75). In keeping with earlier findings, multiple miRNAs testing displayed higher diagnostic accuracy (AUC, 0.92; 84\% sensitivity and $87 \%$ specificity) than assessment of a single miRNA (AUC, 0.84; $78 \%$ sensitivity and $78 \%$ specificity). In another metaanalysis including 103 studies and totaling 3,124 CRC patients and 2,579 healthy individuals, Yan et al. calculated an overall AUC of 0.86 for miRNA testing, reiterating the concept that multiple miRNAs testing may be more effective than analysis of single miRNA (AUC, 0.92 versus 0.81 ; sensitivity, $85 \%$ versus $72 \%$; specificity, $86 \%$ versus 77\%) (77). In 2017, Carter et al. performed another metaanalysis based on 34 studies with 3,454 CRC patients and 2,556 healthy controls (78). In their statistical analysis the authors could identify 17 miRNAs that were upregulated, along with 14 miRNAs which were instead downregulated in CRC patients. A four-miRNA signature, including miR-29a, miR-92a, miR-601 and miR-760, displayed a remarkably high diagnostic performance (AUC, 0.94) for identifying CRC patients, thus persuading the authors to conclude that this four-miRNA signature could even be tested as screening tool in CRC. Nevertheless, an important shortcoming that characterizes all these previous metaanalyses is that the diagnostic efficiency of miRNAs has not been specifically assessed in patients with early CRC stages, whilst patient data have been pooled and analyzed altogether.

As regards other studies, Kanaan et al. demonstrated that an eighth miRNAs panel (miR-532-3p, miR-331, miR-195, miR-17, miR-142-3p, miR-15b, miR-532, and miR-652) was capable to efficiently distinguishing patients with precancerous lesions from healthy controls, displaying an AUC of 0.87 (79). In the study of Zheng et al., including 307 patients with CRC, 164 with colorectal adenoma and 226 healthy controls, a miRNA panel composed of miR-19a-3p, miR-223-3p, miR-92a-3p and miR-422a exhibited good diagnostic accuracy for distinguishing colorectal adenoma from CRC (AUC, 0.89) (80). Fang et al. investigated the expression levels of miR-24, miR-320a and miR-423-5p in plasma samples of 130 healthy controls and in those of 223 patients with colorectal diseases (111 with CRC, 59 with adenoma, 24 with colorectal polyps and 29 with inflammatory bowel disease). The sensitivity of miR24, miR-320a and miR-423-5p for detecting early stage CRC was $77.8 \%, 90.7 \%$, and $88.9 \%$, respectively. The combination of the three miRNAs displayed a cumulative 
AUC of 0.94 (81). Vychytilova-Faltejskova et al. also used a four-miRNAs panel (miR-142-5p, miR-23a-3-p, miR-27a-3p, miR-376c-3p) for distinguishing patients with CRC $(n=203)$ from healthy subjects $(n=100)$, and could demonstrate that this approach efficiently identifies all stages (AUC, 0.92), as well as early stages (I and II) CRC (AUC, 0.88) (82).

Among the miRNAs that have been individually assessed, miR-21 is perhaps the most studied, since is strongly up-regulated in CRC patients and has hence been proposed as both diagnostic and prognostic biomarker (83). In particular, Kanaan et al. reported that plasma miR-21 assessment was an effective strategy for distinguishing CRC patients from healthy controls with $90 \%$ sensitivity, $90 \%$ specificity and 0.91 AUC (84). In the meta-analysis of Zhang and colleagues (85), which included 14 studies and totaled 1,598 patients, the pooled sensitivity, specificity and AUC of circulating miR-21 for diagnosing CRC were found to be as high as $76 \%, 81 \%$ and 0.81 . In a subsequent meta-analysis, based on 9 studies and totaling 746 CRC patients and 476 healthy controls, the pooled sensitivity, specificity and AUC of miR-21 for detecting CRC were $72 \%, 85 \%$ and 0.87 , respectively (86). The subsequent meta-analysis of Peng et al., including 16 studies and involving 1,270 CRC patients and 944 controls (87), revealed that the overall sensitivity, specificity, and AUC of miR-21 for predicting CRC cancer were $64 \%, 85 \%$ and 0.85 , respectively. Unfortunately, no specific subset analysis was conducted for assessing the diagnostic performance of miR-21 in the early stage CRC in any of these meta-analyses.

With the purpose of investigating whether miR-21 could be a useful biomarker for distinguishing benign disease from early stages CRCs, our group has earlier studied miR21 expression in 76 CRC patients and in 20 patients with benign polyps (88). Notably, no significant differences in plasma levels of miR-21 could be observed between early stages CRC patients (stage I and II, $\mathrm{n}=33$ ) and patients bearing benign polyps $(\mathrm{P}=0.85)$. In line with these findings, Kumarswamy et al. showed that miR-21 expression may be enhanced in a vast array of non-neoplastic diseases (89), but it can also be up-regulated in patients with inflammatory diseases (90), and its reliable detection can be impaired be sample hemolysis sample (91) or by contamination with platelet miRNA (92), all factors contributing to lowering its predicated diagnostic performance.

MiR-92a and miR-29a are other interesting molecules, which were found potentially useful for early CRC detection. More specifically, Huang et al. (93) showed that these two miRNAs can efficiently discriminate patients with advanced adenomas from healthy controls with an AUC of 0.77 for miR-29a and 0.75 for miR-92a, respectively. The combination of these two miRNAs yielded even better diagnostic performance, displaying an AUC of 0.88 (83\% sensitivity and $85 \%$ specificity) for detecting CRC, and an AUC of 0.773 ( $73 \%$ sensitivity and $80 \%$ specificity) for identifying patients with advanced adenomas. Interesting evidence has then been recently published by Zanutto et al. (94), who studied plasma miRNAs expression in 60 subjects positive to FIT, and then extended their analysis to an internal validation cohort $(\mathrm{n}=201)$ and to a large external validation cohort ( $\mathrm{n}=1,121$ cases). Overall, a relevant number of miRNAs were found to be significantly deregulated in the first phase of the study (two miRNAs for CRC and six miRNAs for adenomas). In multivariate analysis including sex and age, the AUCs of these signatures were found to be $0.64,0.67$ and 0.68 for low-grade adenoma, high-grade adenoma and CRC, respectively.

In conclusion, although the interesting data emerged from several studies have highlighted the potential clinical usefulness of measuring circulating miRNAs for improving diagnosis and care of CRC patients $(94,95)$, some aspects remain largely undefined, such as diagnostic efficiency and standardization of analytical techniques, lack of a reference standard, increased expression in non-malignant diseases, impact of preanalytical variables, as well as the real possibility of translating this innovative diagnostic approach from the bench to the bedside (96).

\section{Conclusions}

Accumulating evidence suggests that the so-called "liquid biopsy", envisioned as detection and quantification of CTCs and other cancer-related nucleic acids in peripheral blood, may allow earlier diagnosis of CRC combined with lower invasiveness and less patient inconvenience, higher throughput, faster turnaround time, inferior usage of healthcare resources and relatively low cost. Encouraging data have been reported in trials based on CTCs detection, though the sensitivity of the current diagnostic techniques is still perhaps insufficient for enabling early diagnosis of CRC. Among the various biomarkers that can be detected by means of liquid biopsy, SEPT9 methylation displays good diagnostic performance and relatively high cancer detection rate (between $57-64 \%$ in patients with CRC stages 0-I), which would make this test a promising tool to be tested 
in population screening, alone or in combination with more conventional diagnostic investigations. Promising evidence has also been recently published for BCAT1/ IKZF1 methylation. Regarding miRNAs, the combination of some of these biomarkers rather than the assessment of a single miRNA alone seems more effective for efficiently identifying early CRCs, though widespread clinical application is still challenged by a number of preanalytical, analytical and clinical issues.

\section{Acknowledgments}

None.

\section{Footnote}

Conflicts of Interest: The authors have no conflicts of interest to declare.

Etbical Statement: The authors are accountable for all aspects of the work in ensuring that questions related to the accuracy or integrity of any part of the work are appropriately investigated and resolved.

\section{References}

1. Mattiuzzi C, Sanchis-Gomar F, Lippi G. Concise update on colorectal cancer epidemiology. Ann Transl Med 2019. doi: 10.21037/atm.2019.07.91

2. Shaukat A, Mongin SJ, Geisser MS, et al. Long-term mortality after screening for colorectal cancer. $\mathrm{N}$ Engl J Med 2013;369:1106-14.

3. Karamaroudis S, Stamou A, Vorri SC, et al. Monitoring of colonoscopy quality indicators in an academic endoscopy facility reveals adherence to international recommendations. Ann Transl Med 2018;6:263.

4. Fraser CG, Benton SC. Detection capability of quantitative faecal immunochemical tests for haemoglobin (FIT) and reporting of low faecal haemoglobin concentrations. Clin Chem Lab Med 2019;57:611-6.

5. American Cancer Society. American Cancer Society Guideline for Colorectal Cancer Screening. Last accessed, August 18, 2019. Available online: https://www.cancer.org/ cancer/colon-rectal-cancer/detection-diagnosis-staging/ acs-recommendations.html

6. Bénard F, Barkun AN, Martel M, et al. Systematic review of colorectal cancer screening guidelines for average-risk adults: Summarizing the current global recommendations.
World J Gastroenterol 2018;24:124-38.

7. Nishihara R, Wu K, Lochhead P, et al. Long-term colorectal-cancer incidence and mortality after lower endoscopy. N Engl J Med 2013;369:1095-105.

8. Welch HG, Robertson DJ. Colorectal Cancer on the Decline--Why Screening Can't Explain It All. N Engl J Med 2016;374:1605-7.

9. Liu H, Ye D, Chen A, et al. A pilot study of new promising non-coding RNA diagnostic biomarkers for early-stage colorectal cancers. Clin Chem Lab Med 2019;57:1073-83.

10. Montagnana M, Lippi G. Cancer diagnostics: current concepts and future perspectives. Ann Transl Med 2017;5:268.

11. Lauby-Secretan B, Vilahur N, Bianchini F, et al. The IARC Perspective on Colorectal Cancer Screening. N Engl J Med 2018;378:1734-40.

12. Yamada T, Matsuda A, Koizumi M, et al. Liquid Biopsy for the Management of Patients with Colorectal Cancer. Digestion 2019;99:39-45.

13. Wills B, Gorse E, Lee V. Role of liquid biopsies in colorectal cancer. Curr Probl Cancer 2018;42:593-600.

14. Scripcariu V, Scripcariu DV, Filip B, et al. "Liquid Biopsy" - Is it a Feasible Option in Colorectal Cancer? Chirurgia (Bucur) 2019;114:162-6.

15. Nordgård O, Tjensvoll K, Gilje B, et al. Circulating tumour cells and DNA as liquid biopsies in gastrointestinal cancer. Br J Surg 2018;105:e110-20.

16. Lopez A, Harada K, Mizrak Kaya D, et al. Liquid biopsies in gastrointestinal malignancies: when is the big day? Expert Rev Anticancer Ther 2018;18:19-38.

17. Normanno N, Cervantes A, Ciardiello F, et al. The liquid biopsy in the management of colorectal cancer patients: Current applications and future scenarios. Cancer Treat Rev 2018;70:1-8.

18. Pantel K, Speicher MR. The biology of circulating tumor cells. Oncogene 2016;35:1216-24.

19. Rhim AD, Mirek ET, Aiello NM, et al. EMT and dissemination pre-cede pancreatic tumor formation. Cell 2012;148:349-61.

20. Aceto N, Bardia A, Miyamoto DT, et al. Circulating tumor cell clusters are oligoclonal precursors of breast cancer metastasis. Cell 2014;158:1110-22.

21. Giuliano M, Shaikh A, Lo HC, et al. Perspective on circulating tumor cell clusters: why it takes a village to metastasize. Cancer Res 2018;78:845-52.

22. Kalluri R, Weinberg RA. The basics of epithelialmesenchymal transition. J Clin Invest 2009;119:1420-8.

23. Meng S, Tripathy D, Frenkel EP, et al. Circulating tumor 
cells in patients with breast cancer dormancy. Clin Cancer Res 2004;10:8152-62.

24. Baccelli I, Schneeweiss A, Riethdorf S, et al. Identification of a population of blood circulating tumor cells from breast cancer patients that initiates metastasis in a xenograft assay. Nat Biotechnol 2013;31:539-44.

25. Zhang L, Ridgway LD, Wetzel MD, et al. The identification and characterization of breast cancer CTCs competent for brain metastasis. Sci Transl Med 2013;5:180ra48.

26. Alix-Panabières C, Pantel K. Challenges in circulating tumour cell research. Nat Rev Cancer 2014;14:623-31.

27. Krebs MG, Metcalf RL, Carter L, et al. Molecular analysis of circulating tumour cells-biology and biomarkers. Nat Rev Clin Oncol 2014;11:129-44.

28. Cristofanilli M, Budd GT, Ellis MJ, et al. Circulating tumor cells, disease progression, and survival in metastatic breast cancer. N Engl J Med 2004;351:781-91.

29. Kowalik A, lewska M, Góźdź S. Current approaches for avoiding the limitations of circulating tumor cells detection methods-implications for diagnosis and treatment of patients with solid tumors. Transl Res 2017;185:58-84.e15.

30. Abouleila Y, Onidani K, Ali A, et al. Live single cell mass spectrometry reveals cancer-specific metabolic profiles of circulating tumor cells. Cancer Sci 2019;110:697-706.

31. Su W, Yu H, Jiang L, et al. Integrated Microfluidic Device for Enrichment and Identification of Circulating Tumor Cells from the Blood of Patients with Colorectal Cancer. Dis Markers 2019;2019:8945974.

32. Tan $\mathrm{Y}, \mathrm{Wu} \mathrm{H}$. The significant prognostic value of circulating tumor cells in colorectal cancer: A systematic review and meta-analysis. Curr Probl Cancer 2018;42:95-106.

33. Marcuello M, Vymetalkova V, Neves RPL, et al. Circulating biomarkers for early detection and clinical management of colorectal cancer. Mol Aspects Med 2019;69:107-22.

34. Baek DH, Kim GH, Song GA, et al. Clinical Potential of Circulating Tumor Cells in Colorectal Cancer: A Prospective Study. Clin Transl Gastroenterol 2019;10:e00055.

35. Tsai WS, Nimgaonkar A, Segurado O, et al. Prospective clinical study of circulating tumor cells for colorectal cancer screening. J Clin Oncol 2018;36:abstr 556.

36. Jahr S, Hentze H, Englisch S, et al. DNA fragments in the blood plasma of cancer patients: quantitations and evidence for their origin from apoptotic and necrotic cells. Cancer Res 2001;61:1659-65.
37. Danese E, Minicozzi AM, Benati M, et al. Epigenetic alteration: new insights moving from tissue to plasma-the example of PCDH10 promoter methylation in colorectal cancer. Br J Cancer 2013;109:807-13.

38. Danese E, Minicozzi AM, Benati M, et al. Comparison of genetic and epigenetic alterations of primary tumors and matched plasma samples in patients with colorectal cancer. PLoS One 2015;10:e0126417.

39. Petit J, Carroll G, Gould T, et al. Cell-Free DNA as a Diagnostic Blood-Based Biomarker for Colorectal Cancer: A Systematic Review. J Surg Res 2019;236:184-97.

40. Payne SR. From discovery to the clinic: the novel DNA methylation biomarker (m)SEPT9 for the detection of colorectal cancer in blood. Epigenomics 2010;2:575-85.

41. Issa IA, Noureddine M. Colorectal cancer screening: An updated review of the available options. World J Gastroenterol 2017;23:5086-96.

42. Song L, Jia J, Peng X, et al. The performance of the SEPT9 gene methylation assay and a comparison with other CRC screening tests: A meta-analysis. Sci Rep 2017;7:3032.

43. Ahlquist DA, Taylor WR, Mahoney DW, et al. The stool DNA test is more accurate than the plasma septin 9 test in detecting colorectal neoplasia. Clin Gastroenterol Hepatol 2012;10:272-7.e1.

44. Church TR, Wandell M, Lofton-Day C, et al. Prospective evaluation of methylated SEPT9 in plasma for detection of asymptomatic colorectal cancer. Gut 2014;63:317-25.

45. Ding QQ, Zhang H, Xu HC, et al. Study of methylation SEPT9 for colorectal cancer screening in aged population. Chinese Journal of Geriatrics 2015;34:1348-50.

46. deVos T, Tetzner R, Model F, et al. Circulating methylated SEPT9 DNA in plasma is a biomarker for colorectal cancer. Clin Chem 2009;55:1337-46.

47. Grützmann R, Molnar B, Pilarsky C, et al. Sensitive detection of colorectal cancer in peripheral blood by septin 9 DNA methylation assay. PLoS One 2008;3:e3759.

48. He N, Chu WH, Li YQ, et al. Clinical significance of detecting serum methylated Sept9 gene in diagnosis of colorectal cancer. Chinese Journal of Digestion 2014;34:726-31.

49. Jin P, Kang Q, Wang X, et al. Performance of a secondgeneration methylated SEPT9 test in detecting colorectal neoplasm. J Gastroenterol Hepatol 2015;30:830-3.

50. Johnson DA, Barclay RL, Mergener K, et al. Plasma Septin9 versus fecal immunochemical testing for colorectal cancer screening: a prospective multicenter study. PLoS One 2014;9:e98238. 
51. Kang Q, Jin P, Yang L, et al. Significance of Septin9 gene methylation detection of plasma circulation DNA in colorectal cancer screening. Zhonghua Yi Xue Za Zhi 2014;94:3839-41.

52. Li SJ, Liu YG, Wang J. The study of peripheral methylation SEPT9 in screening colorectal cancer. Chin J Gen Surg 2015;124:1756-60.

53. Lofton-Day C, Model F, Devos T, et al. DNA methylation biomarkers for blood based colorectal cancer screening. Clin Chem 2008;54:414-23.

54. Ørntoft M B, Nielsen HJ, Ørntoft TF, et al; Danish Study Group on Early Detection of Colorectal Cancer. et al. Danish Study Group on Early Detection of Colorectal Cancer. Performance of the colorectal cancer screening marker Sept 9 is influenced by age, diabetes and arthritis: a nested case-control study. BMC Cancer 2015;15:819.

55. Song L, Li Y, Jia J, et al. Algorithm Optimization in Methylation Detection with Multiple RT-qPCR. PLoS One 2016;11:e0163333.

56. Tóth K, Sipos F, Kalmár A, et al. Detection of methylated SEPT9 in plasma is a reliable screening method for both left- and right-sided colon cancers. PLoS One 2012;7:e46000.

57. Warren JD, Xiong W, Bunker AM, et al. Septin 9 methylated DNA is a sensitive and specific blood test for colorectal cancer. BMC Med 2011;9:133.

58. Wu D, Zhou G, Jin P, et al. Detection of Colorectal Cancer Using a Simplified SEPT9 Gene Methylation Assay Is a Reliable Method for Opportunistic Screening. J Mol Diagn 2016;18:535-45.

59. Lee JK, Liles EG, Bent S, et al. Accuracy of fecal immunochemical tests for colorectal cancer: systematic review and meta-analysis. Ann Intern Med 2014;160:171.

60. Imperiale TF, Ransohoff DF, Itzkowitz SH, et al. Multitarget stool DNA testing for colorectal-cancer screening. N Engl J Med 2014;370:1287-97.

61. Mitchell SM, Ross JP, Drew HR, et al. A panel of genes methylated with high frequency in colorectal cancer. BMC Cancer 2014;14:54.

62. Mitchell SM, Ho T, Brown GS, et al. Evaluation of Methylation Biomarkers for Detection of Circulating Tumor DNA and Application to Colorectal Cancer. Genes (Basel) 2016;7:E125.

63. Pedersen SK, Baker R, McEvoy A, et al. A two-gene blood test for methylated DNA sensitive for colorectal cancer. PLoS One 2015;10:e0125041.

64. Pedersen SK, Symonds EL, Baker RT, et al. Evaluation of an assay for methylated BCAT1 and IKZF1 in plasma for detection of colorectal neoplasia. BMC Cancer 2015;15:654.

65. Symonds EL, Pedersen SK, Baker RT, et al. A Blood Test for Methylated BCAT1 and IKZF1 vs. a Fecal Immunochemical Test for Detection of Colorectal Neoplasia. Clin Transl Gastroenterol 2016;7:e137.

66. Lin PC, Lin JK, Lin CH, et al. Clinical Relevance of Plasma DNA Methylation in Colorectal Cancer Patients Identified by Using a Genome-Wide High-Resolution Array. Ann Surg Oncol 2015;22 Suppl 3:S1419-27.

67. Lee BB, Lee EJ, Jung EH, et al. Aberrant methylation of APC, MGMT, RASSF2A, and Wif-1 genes in plasma as a biomarker for early detection of colorectal cancer. Clin Cancer Res 2009;15:6185-91.

68. Zhang X, Song YF, Lu HN, et al. Combined detection of plasma GATA5 and SFRP2 methylation is a valid noninvasive biomarker for colorectal cancer and adenomas. World J Gastroenterol 2015;21:2629-37.

69. Bagga S, Bracht J, Hunter S, et al. Regulation by let-7 and lin-4 miRNAs results in target mRNA degradation. Cell 2005;122:553-63.

70. Mitchell PS, Parkin RK, Kroh EM, et al. Circulating microRNAs as stable blood-based markers for cancer detection. Proc Natl Acad Sci USA 2008;105:10513-8.

71. Schwarzenbach H, Nishida N, Calin GA, et al. Clinical relevance of circulating cell-free microRNAs in cancer. Nat Rev Clin Oncol 2014;11:145-56.

72. Khoury S, Tran N. Circulating microRNAs: potential biomarkers for common malignancies. Biomark Med 2015;9:131-51.

73. Byron SA, Van Keuren-Jensen KR, Engelthaler DM, et al. Translating RNA sequencing into clinical diagnostics: opportunities and challenges. Nat Rev Genet 2016;17:257-71.

74. Wang R, Wen $\mathrm{H}, \mathrm{Xu} \mathrm{Y}$, et al. Circulating microRNAs as a novel class of diagnostic biomarkers in gastrointestinal tumors detection: a meta-analysis based on 42 articles. PLoS One 2014;9:e113401.

75. Zeng W, Tu Y, Zhu Y, et al. Predictive power of circulating miRNAs in detecting colorectal cancer. Tumour Biol 2015;36:2559-67.

76. He Y, Lin J, Kong D, et al. Current state of circulating microRNAs as cancer biomarkers. Clin Chem 2015;61:1138-55.

77. Yan L, Zhao W, Yu H, et al. A Comprehensive MetaAnalysis of MicroRNAs for Predicting Colorectal Cancer. Medicine (Baltimore) 2016;95:e2738.

78. Carter JV, Galbraith NJ, Yang D, et al. Blood-based 
microRNAs as biomarkers for the diagnosis of colorectal cancer: a systematic review and meta-analysis. Br J Cancer 2017;116:762-74.

79. Kanaan Z, Roberts H, Eichenberger MR, et al. A plasma microRNA panel for detection of colorectal adenomas: a step toward more precise screening for colorectal cancer. Ann Surg 2013;258:400-8.

80. Zheng G, Du L, Yang X, et al. Serum microRNA panel as biomarkers for early diagnosis of colorectal adenocarcinoma. Br J Cancer 2014;111:1985-92.

81. Fang Z, Tang J, Bai Y, et al. Plasma levels of microRNA-24, microRNA-320a, and microRNA-423-5p are potential biomarkers for colorectal carcinoma. J Exp Clin Cancer Res 2015;34:86.

82. Vychytilova-Faltejskova P, Radova L, Sachlova M, et al. Serum-based microRNA signatures in early diagnosis and prognosis prediction of colon cancer. Carcinogenesis 2016;37:941-50.

83. Toiyama Y, Takahashi M, Hur K, et al. Serum miR-21 as a diagnostic and prognostic biomarker in colorectal cancer. J Natl Cancer Inst 2013;105:849-59.

84. Kanaan Z, Rai SN, Eichenberger MR, et al. Plasma MiR21: a potential diagnostic marker of colorectal cancer. Ann Surg 2012;256:544-51.

85. Zhang $\mathrm{H}, \mathrm{Li} \mathrm{P}, \mathrm{Ju} \mathrm{H}$, et al. Diagnostic and prognostic value of microRNA-21 in colorectal cancer: an original study and individual participant data meta-analysis. Cancer Epidemiol Biomarkers Prev 2014;23:2783-92.

86. Yu W, Wang Z, Shen LI, et al. Circulating microRNA-21 as a potential diagnostic marker for colorectal cancer: a meta-analysis. Mol Clin Oncol 2016;4:237-44.

87. Peng Q, Zhang X, Min M, et al. The clinical role of

Cite this article as: Danese E, Montagnana M, Lippi G. Circulating molecular biomarkers for screening or early diagnosis of colorectal cancer: which is ready for prime time? Ann Transl Med 2019;7(21):610. doi: 10.21037/atm.2019.08.97
microRNA-21 as a promising biomarker in the diagnosis and prognosis of colorectal cancer: a systematic review and meta-analysis. Oncotarget 2017;8:44893-909.

88. Montagnana M, Benati M, Danese E, et al. Plasma Expression Levels of Circulating miR-21 are not Useful for Diagnosing and Monitoring Colorectal Cancer. Clin Lab 2016;62:967-70.

89. Kumarswamy R, Volkmann I, Thum T. Regulation and function of miRNA-21 in health and disease. RNA Biol 2011;8:706-13.

90. Rebane A, Akdis CA. MicroRNAs: Essential players in the regulation of inflammation. J Allergy Clin Immunol 2013;132:15-26.

91. Kirschner MB, Edelman JJ, Kao SC, et al. The Impact of Hemolysis on Cell-Free microRNA Biomarkers. Front Genet 2013;4:94.

92. Cheng HH, Yi HS, Kim Y, et al. Plasma processing conditions substantially influence circulating microRNA biomarker levels. PLoS One 2013;8:e64795.

93. Huang Z, Huang D, Ni S, et al. Plasma microRNAs are promising novel biomarkers for early detection of colorectal cancer. Int J Cancer 2010;127:118-26.

94. Zanutto S, Ciniselli CM, Belfiore A, et al. Plasma miRNAbased signatures in CRC screening programs. Int J Cancer 2019. [Epub ahead of print].

95. Rapado-González Ó, Álvarez-Castro A, López-López R, et al. Circulating microRNAs as Promising Biomarkers in Colorectal Cancer. Cancers (Basel) 2019. doi: 10.3390/ cancers11070898.

96. Danese E, Minicozzi AM, Benati M, et al. Reference miRNAs for colorectal cancer: analysis and verification of current data. Sci Rep 2017;7:8413. 